\title{
La nuova era del trattamento delle acque reflue: l'eMBR è la tecnologia del futuro?
}

\author{
Sara Angela Cantarella ${ }^{1}$ \\ ${ }^{1}$ Affiliation not available
}

\section{Abstract}

\begin{abstract}
Da qualche decennio la comunità scientifica è impegnata nella ricerca delle tecnologie più adatte alla rimozione dei cosiddetti contaminanti emergenti dagli effluenti degli impianti di trattamento delle acque reflue esistenti. Tali impianti, infatti, non sono stati progettati per la rimozione di questi microinquinanti di recente scoperta e non si rivelano quindi efficienti in tal senso. Sono numerose le tecnologie che si stanno studiando a scala di laboratorio e tra queste vi è quella che prevede la combinazione di processi elettrochimici e filtrazione su membrana: la tecnologia eMBR, la quale sembra inserirsi bene nel contesto del trattamento delle acque reflue nei prossimi anni considerando i notevoli risultati ottenuti in termini di rimozione di specifici inquinanti e di upgrade di tecnologie sperimentate.
\end{abstract}

\section{I nuovi orizzonti della ricerca scientifica}

La presenza, ormai consolidata, dei contaminanti emergenti (ECs) nelle acque reflue e nelle acque superficiali e sotterranee è al centro della ricerca scientifica volta, appunto, all'individuazione delle migliori tecnologie in grado di rimuovere tali microinquinanti dalle acque dati i rischi che essi comportano per la salute umana e per gli organismi acquatici.

I convenzionali impianti di trattamento delle acque reflue, ossia quelli attualmente utilizzati, non si sono rivelati in grado di rimuovere tali microinquinanti innanzitutto perché essi non sono mai stati monitorati in quanto non esiste una normativa che li disciplina ${ }^{1}$ e poi perché caratteristica principale di tali ECs è la loro concentrazione molto bassa dell'ordine dei $\mathrm{ng} / \mathrm{L}$ fino a qualche $\mu \mathrm{g} / \mathrm{L}^{2,3,4}$. ${ }^{5}$ hanno infatti riscontrato in uno studio specifico una concentrazione di Carbamazepina negli effluenti degli impianti di trattamento convenzionali dell'ordine di centinaia di $\mathrm{ng} / \mathrm{L}$ e una concentrazione di Diclofenac variabile da "140 ng/L a 1480 ng/L". Si 


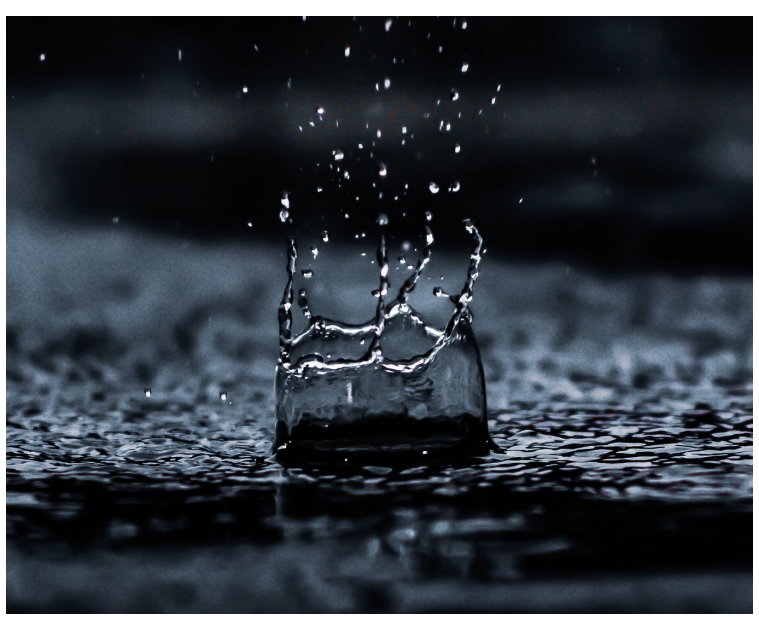

Figure 1: Acqua in un generico corpo idrico

rende, quindi, necessaria l'individuazione di trattamenti specifici che siano in grado di considerare le molteplici proprietà chimiche di cui godono tali composti da cui deriva una variabilità in termini di successo di rimozione del singolo trattamento ${ }^{1}$. In tal senso in alcuni studi sono stati applicati processi di ossidazione con ozono, come nel caso dello studio di ${ }^{6}$ dal quale è però emerso che il raggiungimento di una percentuale soddisfacente di rimozione dei prodotti farmaceutici richiede una concentrazione di ozono elevata e una conseguente formazione rilevante di sottoprodotti, oltre che costi insostenibili. Altri metodi di ossidazione prevedono, invece, una fotocatalisi con $\mathrm{TiO} 2$ e UV/H2O2. Tali metodi consentono di ottenere percentuali di rimozione molto elevate ${ }^{7}$, ma richiedono tempi eccessivamente lunghi e ancora comportano lo sviluppo di sottoprodotti. In alcuni studi si è proposta, quale soluzione di rimozione di composti farmaceutici, il processo di foto-Fenton e di foto-Fenton modificato, in cui si è cercato di ovviare alla necessità di un $\mathrm{pH}$ acido richiesto dal processo di Fenton, aggiungendo agenti complessanti a $\mathrm{pH}$ neutro ${ }^{7}$. I risultati ottenuti sono stati estremamente soddisfacenti, ma anche in questo caso sono i costi elevati il principale fattore limitante. ${ }^{8}$ hanno, invece, realizzato un nuovo adsorbente, ibrido di nanotubi di carbonio granulare (CNT)/allumina che ha mostrato un'elevata rimozione di Carbamazepina e Diclofenac oltre che la possibilità di riutilizzo dell'adsorbente con conseguente riduzione dal punto di vista economico. Soluzioni promettenti sono anche quelle che prevedono l'utilizzo di una membrana di nanofiltrazione e di osmosi inversa. La seconda ha consentito di ottenere una rimozione superiore rispetto alla prima, ma anche consumi energetici maggiori ${ }^{1}$. Sicuramente la tecnologia maggiormente studiata a scala di laboratorio è quella che prevede l'utilizzo dei bioreattori a membrana (MBR) in cui si combinano processi di degradazione biologica con filtrazione su membrane. Tale tecnologia comporta 
una serie di importanti vantaggi rispetto agli impianti convenzionali e in alcuni casi ha consentito la quasi totale rimozione dei microinquinanti, come nel caso dello studio di ${ }^{9}$ in cui l'effluente del bioreattore a membrana è stato sottoposto ad un'unità esterna di ultrafiltrazione seguita da una fase di adsorbimento su carbone attivo granulare (GAC). Tuttavia nell'utilizzo dell'MBR si riscontra ancora una problematica fondamentale che è quella del fouling, ossia dello sporcamento della membrana ${ }^{10}$, inteso sia come incrostazione sulla superficie della stessa che come occlusione, totale o parziale, dei suoi pori. In Figura 2 è riportato l'inquadramento schematico della situazione attuale, con i propri limiti, e degli obiettivi proposti in termini di rimozione di composti farmaceutici.

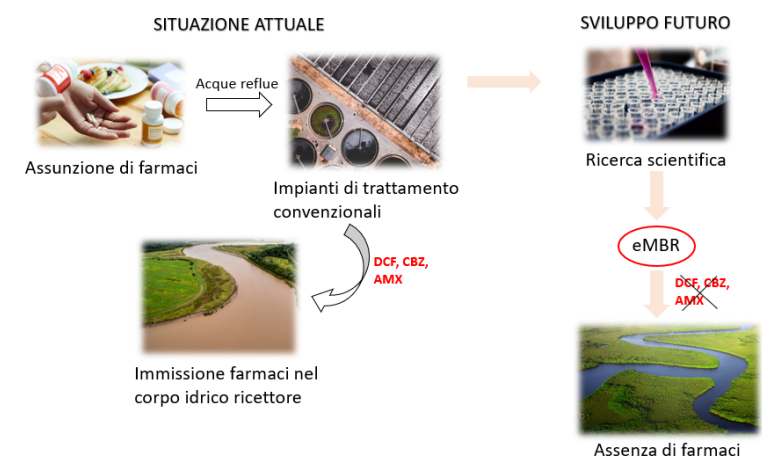

Figure 2: Inquadramento situazione attuale-sviluppo futuro

\section{II ruolo del campo elettrico}

Tra le varie tecnologie, presenti in letteratura, se ne considera in questo elaborato una in particolare: l'eMBR. Si tratta di un bioreattore a membrana (MBR) combinato con processi elettrochimici, il quale ha comportato il potenziamento dell'MBR convenzionale con un'importante riduzione del suo limite principale che è l'elevato tasso di sporcamento della membrana 10,7 , sull'altro una riduzione del consumo energetico apportato normalmente dai processi elettrochimici ${ }^{7}$ ed un incremento delle efficienze di rimozione. L'efficienza di questo tipo di sistema è legata all'immersione all'interno di un bioreattore di una membrana di ultrafiltrazione e di due elettrodi, un anodo e un catodo, (Figura 3) i quali, collegati tramite un filo di rame ad un generatore di corrente, generano un campo elettrico all'interno del bioreattore che induce tre meccanismi elettrochimici principali: elettrocoagulazione, elettrosmosi e elettroforesi ${ }^{10}$. È sostanzialmente a questi tre meccanismi che vanno attribuiti gli straordinari risultati ottenuti con questo tipo di sistema. Grazie, infatti, all'applicazione di un campo elettrico con densità di corrente di $0,5 \mathrm{~mA} / \mathrm{cm}^{2}{ }^{10}$ hanno ottenuto 
una riduzione nella frequenza di lavaggio chimico della membrana rispetto all'MBR convenzionale e questo ha consentito di prolungare la vita utile della membrana stessa. Tale importante risultato è dovuto alla riduzione del fouling della membrana, favorita dal processo di elettrocoagulazione che ha contribuito alla formazione di fiocchi di dimensioni maggiori riducendo quindi la velocità di trasporto attraverso la membrana ${ }^{11}$, dal processo di elettroforesi che ha contrastato la deposizione dei foulants caricati negativamente sulla superficie della membrana spingendoli verso l'anodo ${ }^{12}$, e dal processo di elettrosmosi in cui la parte liquida caricata positivamente è stata spostata verso il catodo e quindi fatta passare attraverso la membrana ${ }^{10}$. Anche in termini di rimozione di inquinanti convenzionali ${ }^{10}$ hanno evidenziato che l'utilizzo di un eMBR consente di ottenere percentuali superiori al $90 \%$, con una rimozione quasi totale di COD, DOC e fosforo e percentuali soddisfacenti nel caso dell'azoto ammoniacale. Sono stati, inoltre, studiati gli effetti di questo sistema in termini di rimozione di prodotti farmaceutici con particolare riferimento alle tre tipologie "maggiormente rilevate negli effluenti degli impianti di trattamento convenzionali": Diclofenac, Carbamazepina e Amoxicillina ${ }^{10}$. In questo caso è all'effetto combinato dell'applicazione del campo elettrico e della filtrazione attraverso membrana che vanno attribuite le elevate efficienze di rimozione ottenute. Il campo elettrico difatti favorisce innanzitutto l'assorbimento di composti farmaceutici disciolti grazie alla formazione di coagulanti da parte del catodo e dell'anodo e poi, grazie al processo di elettrocoagulazione, si ha un incremento delle dimensioni delle molecole dei farmaci che favorisce la loro ritenzione da parte della membrana. Va, infine, sottolineato che nello studio di ${ }^{10}$ si è avuta una particolare attenzione alla riduzione del consumo energetico utilizzando una modalità di funzionamento del campo elettrico intermittente, secondo quanto evidenziato dallo studio $\mathrm{di}^{13}$.

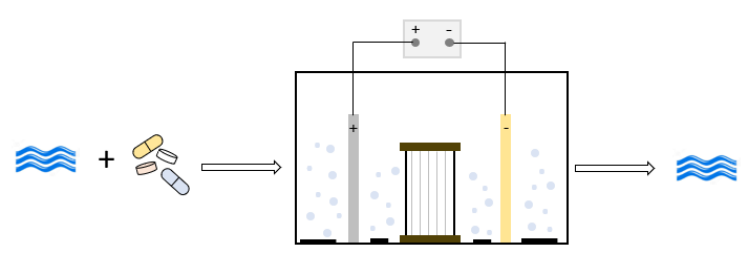

Figure 3: Schematizzazione eMBR

\section{Lo sviluppo futuro}

Gli importanti risultati di cui si è discusso sono, però, attualmente limitati alla ricerca scientifica, ma fanno presagire quelle che sono le potenzialità di questo tipo di sistema. L'eMBR si presenta come un probabile sostituto 
dei convenzionali sistemi di trattamento delle acque ${ }^{10}$ in quanto consente di ottenere un duplice risultato: da un lato elevate efficienze di rimozione, dall'altro il superamento della problematica del fouling, caratteristica dell'MBR. Per quanto riguarda il futuro di questa tecnologia, la comunità scientifica sembra essere interessata ad approfondire le ricerche in tal senso e in alcuni studi si propone di esplorare strade diverse che potrebbero ampliare ulteriormente i confini di questo sistema. Sicuramente il traguardo più immediato sarà l'applicazione della tecnologia al trattamento di acque reflue reali che consentirà di verificare se l'eMBR costituisce effettivamente una svolta nelle modalità di trattamento delle acque reflue.

\section{References}

1.Bolong, N., Ismail, A. F., Salim, M. R. \& Matsuura, T. A review of the effects of emerging contaminants in wastewater and options for their removal. Desalination 239, 229-246 (2009).

2.Hartig, C., Storm, T. \& Jekel, M. Detection and identification of sulphonamide drugs in municipal waste water by liquid chromatography coupled with electrospray ionisation tandem mass spectrometry. Journal of Chromatography A 854, 163-173 (1999).

3.Kasprzyk-Hordern, B., Dinsdale, R. M. \& Guwy, A. J. Multi-residue method for the determination of basic/neutral pharmaceuticals and illicit drugs in surface water by solid-phase extraction and ultra performance liquid chromatography-positive electrospray ionisation tandem mass spectrometry. Journal of Chromatography A 1161, 132-145 (2007).

4.Kasprzyk-Hordern, B., Dinsdale, R. M. \& Guwy, A. J. The removal of pharmaceuticals personal care products, endocrine disruptors and illicit drugs during wastewater treatment and its impact on the quality of receiving waters. Water Research 43, 363-380 (2009).

5.Zhang, Y., Geißen, S. \& Gal, C. Carbamazepine and diclofenac: Removal in wastewater treatment plants and occurrence in water bodies. Chemosphere 73, 1151-1161 (2008).

6.Andreozzi, R. Carbamazepine in water: persistence in the environment ozonation treatment and preliminary assessment on algal toxicity. Water Research 36, 2869-2877 (2002).

7.Klamerth, N., Malato, S., Agüera, A. \& Fernández-Alba, A. PhotoFenton and modified photo-Fenton at neutral $\mathrm{pH}$ for the treatment of emerging contaminants in wastewater treatment plant effluents: A comparison. Water Research 47, 833-840 (2013).

8.Wei, H. et al.. Regenerable granular carbon nanotubes/alumina hybrid adsorbents for diclofenac sodium and carbamazepine removal from aqueous solution. Water Research 47, 4139-4147 (2013). 
9.Wintgens, T., Gallenkemper, M. \& Melin, T. Endocrine disrupter removal from wastewater using membrane bioreactor and nanofiltration technology. Desalination 146, 387-391 (2002).

10.Ensano, B. M. B., Borea, L., Naddeo, V., Luna, M. D. G. de \& Belgiorno, V. Control of emerging contaminants by the combination of electrochemical processes and membrane bioreactors. Environmental Science and Pollution Research 26, 1103-1112 (2017).

11.Hong, H. et al.. Thermodynamic analysis of membrane fouling in a submerged membrane bioreactor and its implications. Bioresource Technology 146, 7-14 (2013).

12.Chen, J. P., Yang, C. Z., Zhou, J. H. \& Wang, X. Y. Study of the influence of the electric field on membrane flux of a new type of membrane bioreactor. Chemical Engineering Journal 128, 177-180 (2007).

13.Borea, L. et al.. Are pharmaceuticals removal and membrane fouling in electromembrane bioreactor affected by current density?. Science of The Total Environment 692, 732-740 (2019).

14.Errami, M. \& Garner, H. A tale of two citations. Nature 451, 397-399 (2008).

15.Zhang. Carbamazepine and diclofenac: Removal in wastewater treatment plants and occurrence in water bodies. Chemosphere 73,

16.Petrovic, M. Analysis and removal of emerging contaminants in wastewater and drinking water. TrAC Trends in Analytical Chemistry 22, 685-696 (2003). 